\title{
Infective Endocarditis on Caseous Calcification of the Mitral Annulus Involving Both the Anterior and Posterior Annulus: A Rare Case Report
}

\author{
Masafumi Ono ${ }^{1}$, Atsushi Mizuno ${ }^{1}$, Keita Masuda ${ }^{1}, K$ Koyu Suzuki ${ }^{2}$, Kohei Abe ${ }^{1}$, \\ Kohei Kawazoe $^{1}$ and Nobuyuki Komiyama ${ }^{1}$
}

\begin{abstract}
:
A 72-year-old man with end-stage renal disease and who was on dialysis was admitted with fever and chills. Two years previously, he had been diagnosed with caseous calcification of the mitral annulus (CCMA). Blood cultures revealed Staphylococcus aureus, and echocardiography revealed vegetation attached to the CCMA lesion, progressing to both the anterior and posterior annulus. Infective endocarditis (IE) was diagnosed and antibiotic (ampicillin) treatment was initiated. Emergent mitral valve replacement was performed after the occurrence of multiple cerebral infarctions. During surgery, we identified vegetation attached to the CCMA lesion. After surgery, the patient showed a good recovery and was discharged. This case demonstrates that IE can be complicated with CCMA.
\end{abstract}

Key words: caseous calcification of mitral annulus, infective endocarditis

(Intern Med 57: 965-969, 2018)

(DOI: 10.2169/internalmedicine.9520-17)

\section{Introduction}

Caseous calcification of the mitral annulus (CCMA) represents a rare evolution of mitral annular calcification (MAC) that occurs due to the caseous transformation of the inner material. Although CCMA is usually considered a benign disease, we herein report a rare case of infective endocarditis (IE) that developed in a patient who had previously been diagnosed with CCMA.

\section{Case Report}

A 72-year-old man with end-stage renal disease and dialysis was admitted due to fever of an unknown origin and chills. He had a long history of hypertension and type 2 diabetes mellitus. We could not find any signs of infection or cerebral stroke. Two years before his admission, transthoracic echocardiography revealed a calcified mass at the base of the posterior leaflet of the mitral valve, which was diagnosed as CCMA (Fig. 1). In comparison to the previous findings, transthoracic echocardiography at admission revealed an enlargement of the CCMA lesion, which had progressed to both the anterior and posterior annulus, but no obvious vegetation. Because blood cultures at admission yielded methicillin-susceptible Staphylococcus aureus (MSSA), we performed a transesophageal echocardiography, which revealed a $12-\mathrm{mm} \times 8-\mathrm{mm}$ area of mobile vegetation attached to the base of the posterior mitral valve leaflet (Fig. 2).

The patient was diagnosed with infective endocarditis with MSSA and was treated with ampicillin after high sensitivity to ampicillin was confirmed. The patient experienced a convulsion on day 5; thus, we performed magnetic resonance imaging, which revealed multiple cerebral infarctions without hemorrhage. We therefore performed emergent mitral valve replacement.

The posterior annulus of the mitral valve showed thickening due to caseous calcification. The vegetation was attached to the posterior mitral annulus of the second leaflet (Fig. 3A). When the mitral annulus was incised, pasty materials flowed out from the incisions (Fig. 3B). We used an

${ }^{1}$ Cardiovascular Center, St. Luke's International Hospital, Japan and ${ }^{2}$ Department of Pathology, St. Luke's International Hospital, Japan Received: May 17, 2017; Accepted: July 28, 2017; Advance Publication by J-STAGE: December 21, 2017

Correspondence to Dr. Atsushi Mizuno, atmizu@gmail.com 

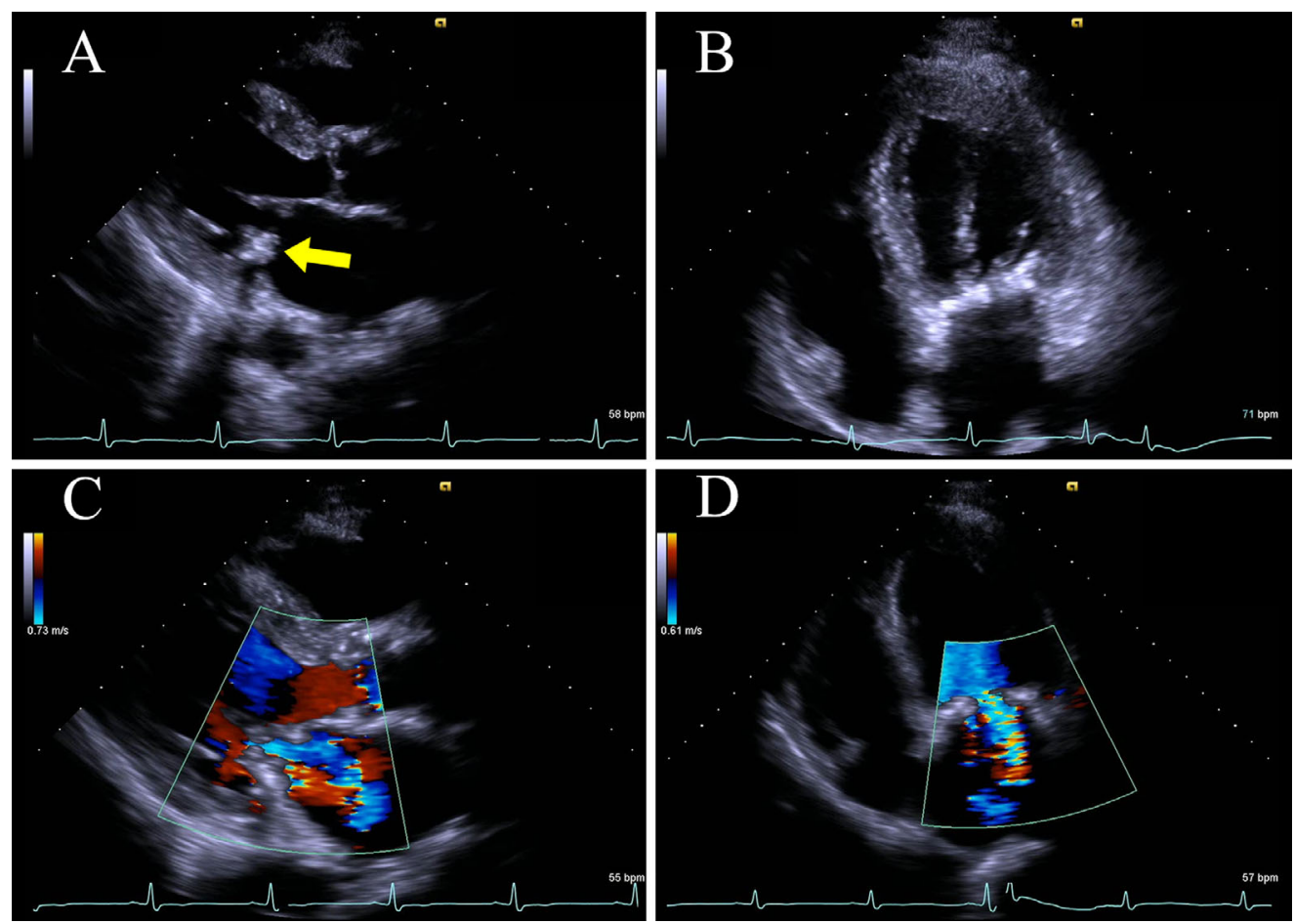

Figure 1. Transthoracic echocardiography with a long-axis parasternal view and a 4-chamber apical view (A and B) shows a rounded, calcified mass at the base of the posterior leaflet of the mitral valve (yellow arrow). Color Doppler $(C$ and $D)$ shows mild regurgitation of the mitral valve.
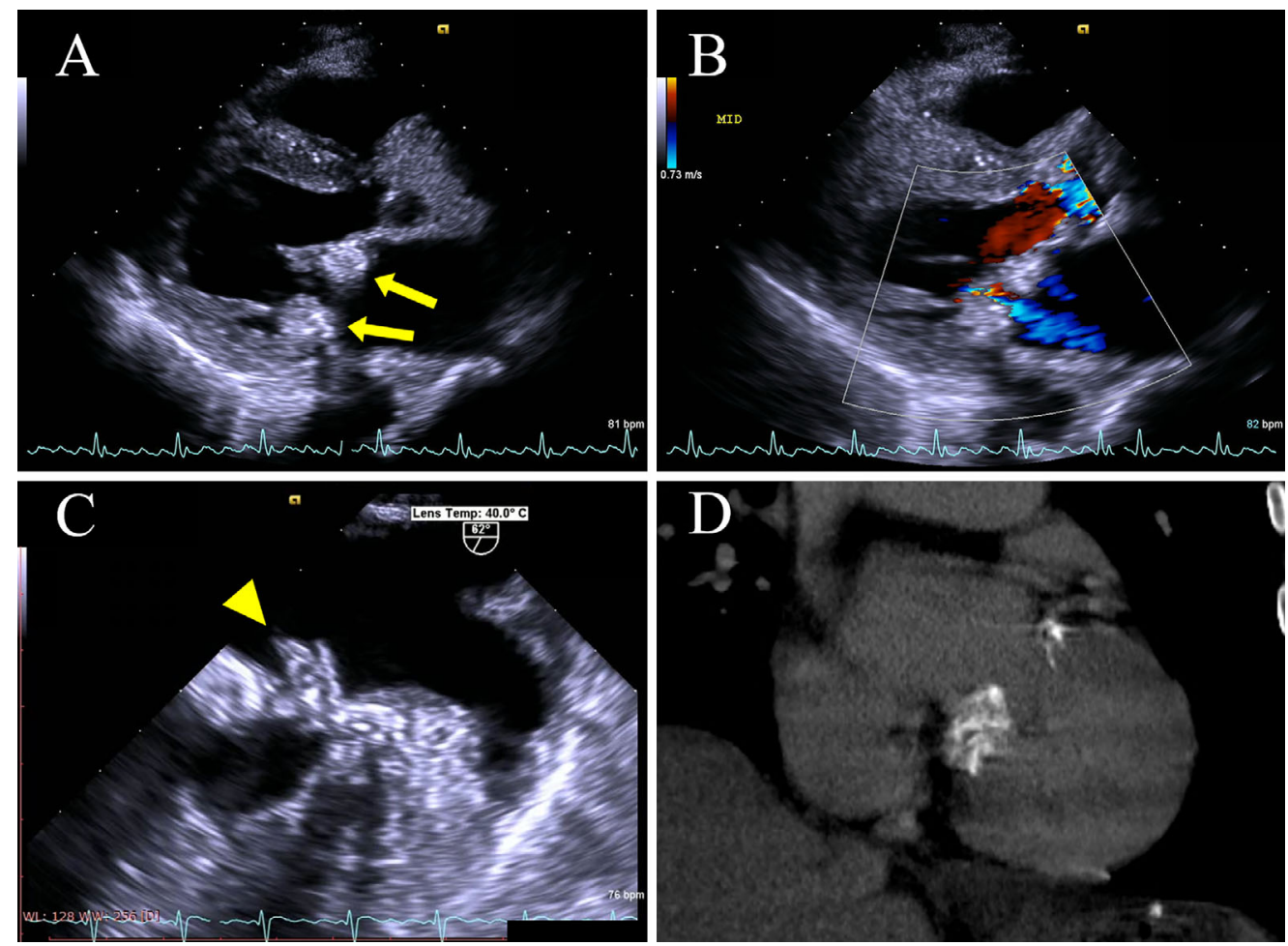

Figure 2. Transthoracic echocardiography with a long-axis parasternal view after admission (A) shows advanced CCMA progressing on both the posterior and anterior annulus of the mitral valve (yellow arrow). The features of CCMA, such as central echolucency resembling liquefaction, are clearer than they were in the previous test. Color Doppler (B) shows mild regurgitation of the mitral valve, similar to the previous echocardiogram. Transesophageal echocardiography $(C)$ shows vegetation attached to the base of the posterior mitral valve leaflet (P2) (yellow arrowhead). Non-contrast computed tomography (D) confirms the presence of diffuse calcification of the mitral valve. 


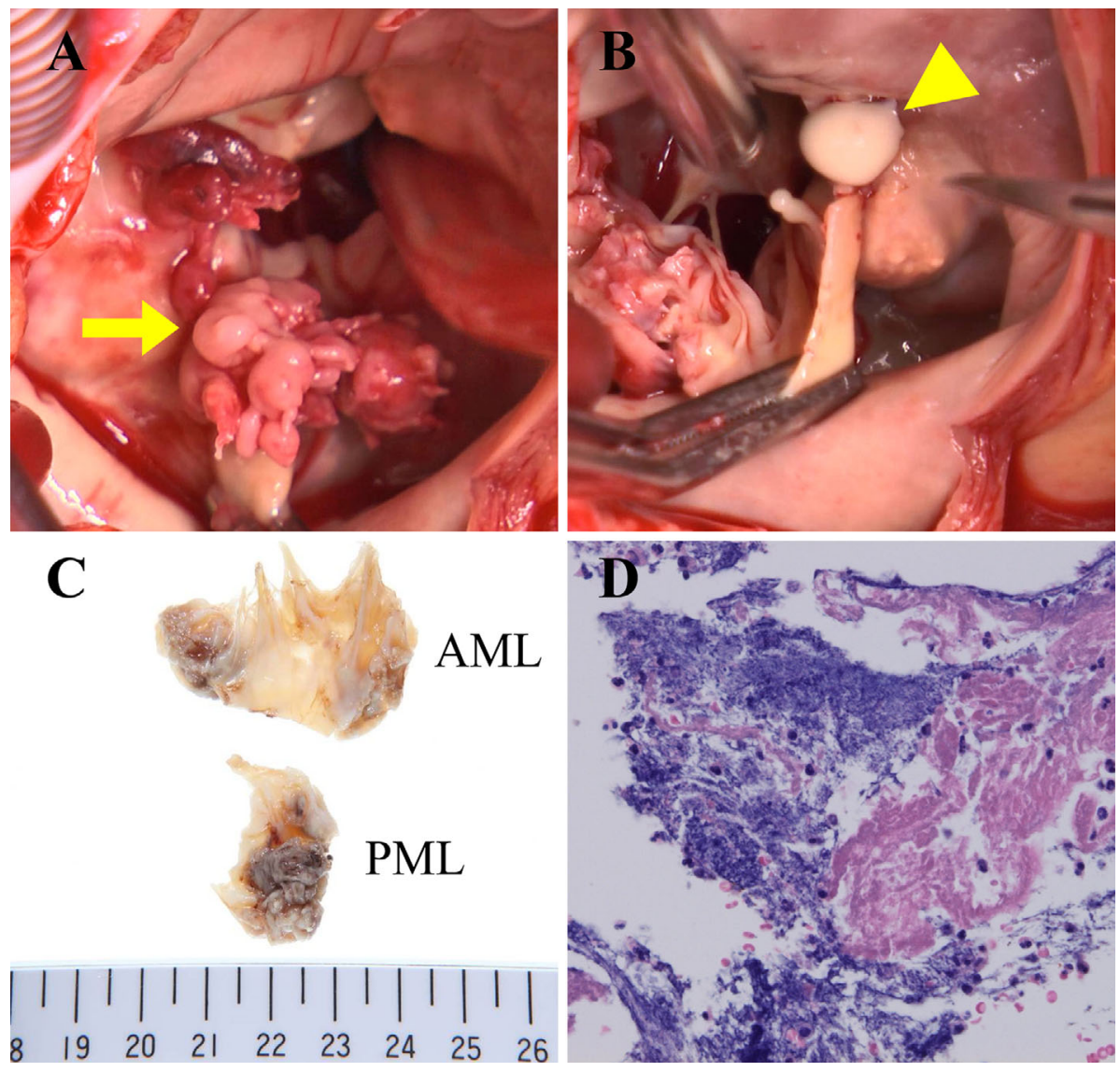

Figure 3. An intraoperative view (A) shows the vegetation of bacterial endocarditis attached to the base of the posterior mitral valve leaflet (P2). Caseous calcification covers the entire circumference of the mitral annulus. A white pasty material flows out from the incised mass of the mitral annulus (B). The examination of tissues of the mitral valve leaflet $(C$ and $D)$ reveals vegetation of infective endocarditis with neutrophilic infiltration, vascularization and bleeding.

On-X bileaflet mechanical heart valve (On-X Life Technologies, Austin, USA) to replace the mitral valve. Cultures of both the resected mitral leaflets and the pasty materials in the CCMA lesion yielded MSSA.

The mitral valve leaflet tissue, which was incised for a pathological examination (Fig. 3C and D), showed vegetation composed of fibrin, necrotic debris, neutrophilic infiltration, inflammatory granulation tissue and a bacterial colony. Although the patient required management for heart failure, he showed a good recovery after surgery and was discharged on day 39.

\section{Discussion}

We described a case of infective endocarditis that developed in a patient who was previously diagnosed with CCMA, and which was confirmed by the examination of a tissue specimen. Surgery, which was performed immediately after the determination, achieved a successful clinical outcome.

CCMA, which is a relatively rare condition, is found in $006-0.07 \%$ of the population (1-3). MAC, a more common condition which is defined as the chronic degeneration of the fibrous ring of the of the mitral valve, occurs in approxi- mately $10.6 \%$ of the population (3). CCMA tends to occur in older patients (mean age: 69 years) and is associated with hypertension and end-stage renal disease (particularly in those on hemodialysis), which was comparable to our case (3-5). The pathogenesis of caseous calcification remains unclear. Hypercholesterolemia and the dissolution of lipidladen macrophages may be implicated in liquefaction necrosis (3).

CCMA more frequently develops in the posterior annulus of the mitral valve; there are few reported cases of CCMA at the anterior annulus. Harpaz et al. reported that in a series of 19 patients with CCMA, there was only one patient in whom both the anterior and posterior annulus (similarly to our case) were involved (1). Similarly, Deluca et al. reported that only 2 of 14 cases showed CCMA at both the anterior and posterior annulus, and in all other cases, the CCMA lesion was located at the posterior annulus alone (3). The reason why CCMA frequently occurs at the posterior annulus has not been reported. It may be related to the fact that MAC is more commonly observed at the posterior annulus than at the anterior annulus (6). In our case, CCMA initially developed at the posterior leaflet and extended to the anterior leaflet at admission.

The histopathological features of CCMA include the pres- 
ence of pasty material in the central region, a dense, amorphous and basophilic substance, sparse histiocytes and scattered calcifications that were prevalently located in the peripheral regions (7).

Harpaz et al. reported that transthoracic echocardiography was sufficient for making a correct diagnosis of CCMA. The typical echocardiographic features included a round, large, echo-dense mass with smooth borders and a central area of echolucency, resembling liquefaction $(1,3)$. Computed tomography (CT) may contribute to a diagnosis of CCMA. On CT, CCMA is reported to appear as a round or semilunar sharply marginated mass with a hypo-dense or hyperdense center and a calcified peripheral rim (8). The absence of enhancement after the administration of contrast agent would be useful for differentiating CCMA from other cardiac masses, such as tumors (9). In our case, previous echocardiography clearly showed an echo-dense mass and that was sufficient for a diagnosis of CCMA; the diagnosis was confirmed by CT.

CCMA is usually a benign lesion and rarely causes mitral regurgitation, mitral stenosis or arrhythmia (such as atrioventricular block). Thus, in many cases, surgery - or other forms of therapeutic intervention - is not required (10).

On the other hand, some reports have revealed that CCMA may cause cerebral infarction. A review by Dietl et al. reported that patients with CCMA had significantly higher rates of cerebral infarction in comparison to patients with MAC alone (19.2\% vs. $11.8 \%)$ (11). Some of the mechanisms through which CCMA may cause cerebral infarction are as follows: (1) spontaneous fistulization and embolization of caseous material that may leak directly into the left atrium or ventricle; (2) embolization of caseous necrotic debris from the CCMA lesion; and (3) embolization of calcium and cholesterol particles. In our case, multiple cerebral infarctions occurred; however, we considered infective embolism from IE to be the cause of the patient's cerebral infarctions due to several reasons: there was no obvious change in the formation of CCMA before and after cerebral infarction, the smooth surface, the fact that no fistulization or cavity on the CCMA lesion were observed during surgery, and the presence of mobile vegetation (of $>10 \mathrm{~mm}$ in size) due to $S$. aureus, all of which are known as risk factors for infective infarction in patients with IE (12).

There is only one other reported case of IE with CCMA in which vegetation and CCMA were simultaneously confirmed by transesophageal echocardiography on admission (13). Thus, to our knowledge, this is the first report in which IE developed in a patient who was previously diagnosed with CCMA. In addition, to our knowledge, this is the first case report to pathologically demonstrate that IE can occur on CCMA.

Considering the pathophysiology and the mechanisms of IE of MAC, it is reasonable to consider that a CCMA lesion can develop infective endocarditis. The mechanism through which MAC is infected remains unclear. Pressman et al. reported that 16 of 22 patients with vegetation on MAC were infected with $S$. aureus (14). They mentioned that $S$. aureus adhered to the endocardium by a different mechanism from streptococcal species, and that it could have more affinity for osteoblastic changes, which would be caused inside MAC. In our case, the infecting organism was also $S$. aureus, thus a similar process might have occurred in the CCMA lesion. Furthermore, the protrusion of annular calcium might have resulted in turbulent blood flow and ulceration of the overlying leaflet, and the formation of a thrombus at the edge of the area of ulceration could provide a nidus for infection $(15,16)$.

Based on the findings of the present case, if a patient with CCMA has a fever of unknown origin, as well as the presence of MAC, which is a strong risk factor for IE, the possibility of IE must be excluded. The echocardiographic diagnosis of IE with MAC is difficult because the vegetation is masked by artifacts and shadowing from the calcification (15). The diagnosis of IE with CCMA by echocardiography could be more difficult than IE with MAC, because of the large mass mimicking a vegetation and progressed calcification. Blood culturing and echocardiography are needed more frequently in comparison to cases without CCMA.

The patient who experienced multiple cerebral infarctions made a successful recovery after surgery. In the present case, none of the observations were absolute indicationsaccording to recently published guidelines (17-19) - for early surgery before the occurrence of cerebral infarction. However, the appropriate timing of surgery could have been earlier because, as previously mentioned, there were several risk factors for cerebral infarction. The timing of surgery should be decided on an individual basis according to the risks, and the risks of cerebral infarction associated with MAC or CCMA should be emphasized when considering the indications for surgery in patients with IE.

In the present case, blood cultures from both the incised tissue of the mitral leaflets and the pasty materials in the CCMA lesion were positive. This helped us to diagnose IE with CCMA. Whether the bacteria penetrated the CCMA lesion from the surface, or occurred inside the material of the CCMA lesion and progressed outward, is still unknown.

\section{Conclusion}

We reported a rare case of infective endocarditis that developed in a patient previously diagnosed with CCMA. We emphasize the importance of the careful observation of patients with CCMA. When performing procedures that increase the risk of infection in CCMA patients, periprocedural antibiotics might also be considered-similarly to patients with MAC.

The authors state that they have no Conflict of Interest (COI).

\section{References}

1. Harpaz D, Auerbach I, Vered Z, Motro M, Rosenblatt S. Caseous 
calcification of the mitral annulus: a neglected, unrecognized diagnosis. J Am Soc Echocardiogr 14: 825-831, 2001.

2. Kronzon I, Winer HE, Cohen ML, Cohen A. Sterile, caseous mitral anular abscess. J Am Coll Cardiol 2: 186-190, 1983.

3. Deluca G, Correale M, Ieva R, Salvatore B, Del Gramenzi S, Di Biase M. The incidence and clinical course of caseous calcification of the mitral annulus: a prospective echocardiographic study. J Am Soc Echocardiogr 21: 828-833, 2008.

4. Elgendy IY, Conti CR. Caseous calcification of the mitral annulus: A review. Clin Cardiol 36: 27-31, 2013.

5. Akram M, Hasanin AM. Caseous mitral annular calcification: Is it a benign condition? J Saudi Hear Assoc 24: 205-208, 2012.

6. Abramowitz Y, Jilaihawi H, Chakravarty T, Mack MJ, Makkar RR. Mitral annulus calcification. J Am Coll Cardiol 66: 1934-1941, 2015.

7. Di Bella G, Carerj S, Andò G, et al. Cardiac imaging in the evaluation of mitral annulus caseous calcification. Int $\mathrm{J}$ Cardiol 113 30-31, 2006.

8. Vanovermeire OM, Duerinckx AJ, Duncan DA, Russell WG. Caseous calcification of the mitral annulus imaged with 64-slice multidetector $\mathrm{CT}$ and magnetic resonance imaging. Int $\mathrm{J}$ Cardiovasc Imaging 22: 553-559, 2006.

9. Mallat $\mathrm{N}$, Limeme M, Zaghouani $\mathrm{H}$, et al. Caseous calcification of the mitral annulus on MDCT: a rare intracardiac mass. Acta Radiol Short Reports 2: 204798161350217, 2013.

10. Wehman B, Dawood M, Ghoreishi M, et al. Surgical management of caseous calcification of the mitral annulus. Ann Thorac Surg 99: 2231-2233, 2015.

11. Dietl CA, Hawthorn CM, Raizada V. Risk of cerebral embolization with caseous calcification of the mitral annulus: review article. Open Cardiovasc Med J 10: 221-232, 2016.

12. Pergola V, Rosenberg V, Casalta J, et al. Risk of embolism and death in infective endocarditis: prognostic value of echocardiography a prospective multicenter study. Circulation 112: 69-75, 2005.

13. Capin E, Leon D, Rodriguez ML, et al. Infective endocarditis and caseous calcification of the mitral annulus: the odd couple. Arch Cardiol Mex 84: 100-101, 2014.

14. Pressman GS, Rodriguez-ziccardi $\mathrm{M}$, Gartman $\mathrm{CH}$, et al. Mitral annular calcification as a possible nidus for endocarditis: a descriptive series with bacteriological differences noted. J Am Soc Echocardiogr 30: 572-578, 2017.

15. Tsunekawa T, Kobayashi J, Tagusari $\mathrm{O}$, et al. Infective endocarditis and pseudoaneurysm: fatal complications of mitral annular calcification. Ann Thorac Surg 81: 1881-1882, 2006.

16. Hospital TG, Hospital M. Bacterial endocarditis of the mitral valve associated with annular calcification. 119: 323-326, 1978.

17. Baddour LM, Wilson WR, Bayer AS, et al. Infective endocarditis in adults: diagnosis, antimicrobial therapy, and management of complications. Circulation, 2015 (Epub ahead of print).

18. Habib H, Lancellotti P, Antunes MJ, et al. 2015 ESC Guidelines for the management of infective endocarditis: The task force for the management of infective endocarditis of the European Society of Cardiology (ESC) endorsed by: European Association for Cardio-Thoracic Surgery (EACTS), the European Association of Nuclear Medicine (EANM). Eur Heart J 36: 3075-3128, 2015.

19. JCS Joint Working Group. Guidelines for the prevention and treatment of infective endocarditis (JCS 2008). Circ J 72: 1253-1318, 2008 .

The Internal Medicine is an Open Access article distributed under the Creative Commons Attribution-NonCommercial-NoDerivatives 4.0 International License. To view the details of this license, please visit (https://creativecommons.org/licenses/ by-nc-nd/4.0/).

(C) 2018 The Japanese Society of Internal Medicine

Intern Med 57: 965-969, 2018 